\title{
The Self and Social Judgment: Effects of Affective Reaction and "Own Position" on Judgments of Unambiguous and Ambiguous Information About Others
}

\author{
Alan J. Lambert \\ University of Illinois at Urbana-Champaign
}

\author{
Douglas H. Wedell \\ University of South Carolina
}

\begin{abstract}
Ss who differed in the extremity of self-definition ("own position") with respect to a given trait (sociability, independence, or patience) made trait and evaluative judgments of behavioral stimuli that varied in their descriptive implications for that trait. Across 4 experiments, individual differences in trait ratings of unambiguous information were mediated largely by differences in $\mathrm{Ss}^{*}$ affective reactions to these stimuli rather than by direct use of own position as a judgmental anchor. When the target information was ambiguous, however, own position influenced trait judgments independently of Ss' affective reactions to these stimuli. These latter effects were moderated by either encoding or informational mechanisms. A theoretical framework is presented that accounts for these results and predicts how effects of self-knowledge on judgments of others should vary across different trait dimensions.
\end{abstract}

The idea that one's own characteristics can influence perceptions of others can be traced as far back as Freud (1896/1956), who argued that people may sometimes "project" their own undesirable traits onto others. Within modern social psychology, this notion is articulated in the generally accepted principle that social judgment is determined both by the features of the stimulus being judged and by the characteristics of the perceiver (Heider, 1958).

The effects of perceiver variables on judgments of others, and how these effects may vary depending on the type of target information being judged, can be assessed empirically by varying the characteristics of both factors in the same design (cf. Park \& Hahn, 1988). This approach has been explored most thoroughly in the attitude literature, in which researchers have often examined the relation between subjects' own attitudes and their judgments of specific opinion statements. Judgments of such statements have typically been found to be positively or negatively related to subjects' own attitudinal position, depending on the similarity of the target statement to the position of the judge (e.g., Sherif \& Hovland, 1961). (For reviews of this literature, see Eiser, 1986; Petty \& Cacioppo, 1981.)

In light of this large body of research, it is surprising that

This research was financially supported in part by a Nancy Hirschberg Memorial Award to Alan J. Lambert and by National Institute of Mental Health Grant MH3-8585, BSR, to Robert S. Wyer.

Special appreciation is expressed to Robert $S$. Wyer for his valuable insights and clarification of many of the ideas in this article. Appreciation is also extended to Stephan Ahadi, Ulf Bockenholt, Lee Budesheim, Robert Fuhrman, David Funder, Tim Harper, and Fritz Strack for excellent comments and criticisms of earlier drafts. Finally, we wish to acknowledge the members of the Illinois Social Cognition Group for their insights into the interpretation of the results of these studies.

Correspondence concerning this article should be addressed to Alan J. Lambert, Department of Psychology, University of Illinois, 603 East Daniel Street, Champaign, Illinois 61820 . relatively few studies have used a similar two-way (Target $x$ Perceiver) design to examine the possible effects of subjects' own trait position on the way that the trait characteristics of others are judged. Instead, most research in the trait domain has been conducted within the false consensus paradigm, in which subjects are asked to infer the traits of other persons (e.g., "college students") about whom little or no trait-relevant information is provided. (For a review, see Marks \& Miller, 1987) Of those few investigations that have varied trait-relevant features of both target and perceiver in the same design, results across studies have been somewhat inconsistent (e.g., Markus \& Fong, 1979; Markus, Smith, \& Moreland, 1985; Park \& Hahn, 1988). ${ }^{1}$

The goal of the present research is to gain further understanding of the cognitive and affective processes by which differences in self-definition with respect to a given trait dimension (hereinafter, "own position") might influence judgments of social targets along that dimension. Because previous studies conducted in other research domains have provided evidence that the processes underlying judgments of unambiguous and ambiguous information may differ (e.g., Herr, Sherman, \& Fazio, 1983), we consider the theoretical issues for each class of stimuli separately.

\section{Judgments of Unambiguous Information}

Suppose that subjects were asked to form sociability judgments of behavioral descriptions in which implications for that

\footnotetext{
${ }^{1}$ For example, whereas some studies (e.g., Markus \& Fong, 1979) in which unambiguous information about others is presented for judgment have produced results that are quite consistent with the pattern of assimilation/contrast predicted by social judgment theory (Sherif \& Hovland, 1961), other studies (e.g., Markus, Smith, \& Moreland, 1985; Park \& Hahn, 1988) have found little or no contingency between the self and direct trait ratings of this kind of information. A possible reason why differences in self-definition do not always lead to differences in the trait ratings of unambiguous information about others is discussed later in this article.
} 
trait are either unambiguously low (e.g., "rarely talks to others in the hallway") or high (e.g. "goes to three parties a week"). Some researchers (e.g., Markus et al, 1985; Markus \& Zajonc, 1985) have suggested that the self should have relatively little effect on judgments of target information that has clear and unambiguous implications for the trait dimension of judgment. Therefore, it is conceivable that subjects' own sociability would be unrelated to how such behaviors are judged.

Research from the attitude literature provides evidence, however, that the effects of the self are not necessarily eliminated when the target information has unambiguous implications for the dimension of judgment. Indeed, strong and reliable effects of subjects' own attitude on judgments of unambiguously pro or anti statements have often been demonstrated in the social judgment literature (Sherif \& Hovland, 1961). To the extent that the processes underlying attitudinal judgments would generalize to the trait domain, there appears to be at least two mechanisms by which the self could influence judgments of unambiguous behaviors.

\section{Self-as-Anchor Effects}

The notion that judgments of attitudinal opinions may be influenced by the extremity of own position is articulated by at least three theories of attitudinal judgment: (a) adaptation-level theory (Helson, 1964), (b) social judgment theory (Sherif \& Hovland, 1961), and (c) the variable perspective approach (Upshaw, 1969). Although these theories differ in some important respects, all three share the basic assumption that it is the extremity of the judge's own position per se that is an important determinant of how the stimulus is judged (cf. Judd \& Harackiewicz, 1980).

Perhaps the best known of these theories is Sherif and Hovland's (1961) social judgment theory. Two aspects of this formulation are important to note. First, the theory describes a rather direct effect of the self as a judgmental anchor in which (a) judgments of statements close to the anchor are displaced toward own position (assimilation) and (b) judgments of statements further removed from the anchor are displaced away (contrast). Second, although later versions of the theory (e.g., Sherif \& Sherif, 1967) suggest that ego involvement (i.e., importance) of the attitude domain to the self may be necessary for such judgmental effects to occur, the theoretical mechanisms postulated to be responsible for assimilation and contrast do not involve the judge's evaluative reaction to the stimulus to be judged. Indeed, Sherif and Hovland proposed that the effects demonstrated in the attitude domain reflect the same processes that guide judgments of evaluatively neutral stimuli, such as judgments of the heaviness of lifted weights (Sherif, Taub, \& Hovland, 1958).

Social judgment theory has received some support in both the psychophysical and attitude domain, although the evidence for assimilation effects appears to be much weaker than for contrast effects (cf. Eiser \& van der Plight, 1984). Similar principles might apply to the trait domain. For example, subjects who consider themselves to be highly sociable could theoretically use their own sociability as a standard of comparison when judging the sociability of others, resulting in a contrast effect.

\section{Affect-Based Polarization Effects}

Although it is well-documented that subjects who vary in their attitudes may judge the same opinion very differently, accentuation theory (Eiser \& Stroebe, 1972; Tajfel, 1957) suggests that this effect may only indirectly involve differences in subjects' attitudinal positions per se. Instead, perceivers who judge the attitudinal opinion held by another person are likely to use their affective (evaluative) reaction ${ }^{2}$ to the stimulus as an additional informational cue to judge it along a descriptive, pro-anti dimension. For example, a pro-feminist's unfavorable reaction to an anti-feminist statement may be used as additional information that the statement indeed represents an anti position, thus polarizing judgments further in the anti direction. Similarly, a strong positive affective reaction to a pro-feminist statement should result in accentuating judgments of that statement in the pro direction.

The strength or intensity of affective reactions to attitudinal opinions has been shown to correlate positively with the extremity of the judge's own opinion (Judd \& Johnson, 1984; Suchman, 1950). Those who hold more extreme positions should therefore show greater accentuation of judgment, leading (for example) extreme pro-feminists to judge pro-feminist and antifeminist statements as more pro and more anti than moderate pro-feminists (Judd \& Harackiewicz, 1980). (For a more detailed account of this process, see Eiser, 1986; Eiser \& Stroebe, 1972.)

These accentuation effects strongly resemble the pattern of assimilation and contrast predicted by social judgment theory (Sherif \& Hovland, 1961), but the theories differ in at least two important ways. First, social judgment theory proposes a direct influence of the judge's own position, but accentuation theory proposes indirect effects of own position, as mediated by the judge's affective reactions to the target. Second, accentuation theory predicts systematic effects of manipulating the salience of affective reactions to the stimuli being judged, whereas the assimilation and contrast model does not (cf. Judd \& Harackiewicz, 1980). In the attitude literature, tests designed to distinguish between these two theories have generally supported accentuation theory over social judgment theory (e.g., Eiser, 1986; Judd \& Johnson, 1984), but the processes postulated by the two theories could in principle have additive effects. For example, extreme pro-feminist subjects could judge an anti-feminist statement as extremely anti by virtue of both their extreme affective reaction to it (accentuation) as well as their use of own position as a judgmental anchor (contrast). However, the extent to which either of these processes operate, if at all, in the trait domain is presently unclear. A general aim in the present research was therefore to tease apart these different sources of individual differences in trait judgments of others.

\footnotetext{
${ }^{2}$ In this article, we use both affective reaction and evaluative reaction interchangeably to refer to the subjective favorableness of the target stimulus during information processing. Although the theoretical distinction between one's affective reaction to a target stimulus and one's evaluation of that stimulus is important (Wyer \& Srull, 1989), this issue is beyond the scope of the current article.
} 


\section{Judgments of Ambiguous Information}

Suppose that subjects were asked to judge the sociability of the behavioral description, "installed a telephone answering machine in his apartment." This statement could be considered to be ambiguous with respect to sociability because it can imply either high or low levels of that trait. For example, it could be interpreted as reflecting an effort to (a) avoid missing invitations to social engagements or (b) refrain from having to converse with others. Theoretical and empirical work suggests two basic types of processes that might mediate judgments of this kind of information.

\section{Encoding Effects}

According to research in the priming literature (e.g., Herr et al., 1983; Higgins, Rholes, \& Jones, 1977; Srull \& Wyer, 1980), trait judgments of ambiguous behaviors may be conceptualized in terms of a process in which the stimuli are categorized with the (applicable) concept that is most accessible at the time of judgment. Although category accessibility can and has been manipulated experimentally, certain traits may also be chronically accessible over time and across a variety of situations (Kelly, 1955). The chronic accessibility of a trait concept (as measured by the frequency and primacy that the concept is used to refer to others) has been shown to produce encoding effects on the judgments of ambiguous stimuli that are additive to the effects of experimental manipulation of its accessibility (Bargh, Bond, Lombardi, \& Tota, 1986).

Although many factors could lead to the chronic accessibility of a given trait concept (Higgins, 1989; Higgins \& King, 1981), there is some evidence that individual differences in chronic accessibility are related to the extremity of own position along that dimension. For example, several studies have found a positive relation between extremity of own position and the efficiency with which trait information about the self and others is processed (e.g., Bargh, 1982; Markus, 1977; Markus \& Smith, 1981). ${ }^{3}$ Such effects theoretically reflect the chronic or longterm accessibility of such traits in memory (Bargh, 1984, 1989; Higgins \& King, 1981).

In light of these and other findings, Higgins, King, and Mavin (1982) have suggested that a similarity between judgments of the self and others may be attributable, at least in part, to differences in chronic accessibility. For example, highly sociable subjects who are asked to form sociability judgments of an ambiguous behavior may be more likely to encode this statement as sociable relative to subjects whose own position is less extreme (and therefore, for whom that trait concept may be less accessible). As Higgins et al. (1982) were careful to note, however, this process does not necessarily involve direct access of self-relevant knowledge. That is, similarity in self-other judgments may simply reflect a tendency for some individuals to use certain constructs in processing information about people in general, including the self. (For alternate conceptualizations of these and other related issues, see Bargh, 1984; Markus \& Smith, 1981; see also Higgins \& Bargh, 1987; Segal, 1988.)

\section{Informational Effects}

An alternative to an encoding explanation is that subjects who differ in their own position also differ in the kinds of information that they would draw upon to judge an ambiguous stimulus. At least two types of information might be used. First, judges may use knowledge about their own characteristics as a direct basis to infer the attributes of others. That is, in the absence of diagnostic information, people may have a tendency to assume that others have characteristics that are similar to their own for either cognitive or motivational reasons (cf. Marks \& Miller, 1987). Second, to the extent that people typically associate with individuals whose characteristics are similar to their own (Berscheid \& Walster, 1978; Newcomb, 1961), subjective beliefs about the characteristics of others (i.e., subjective base rates) might also be used as a source of information separate from the direct use of self-information.

The proposed encoding and information-based processes are similar in that (a) both would typically result in a positive correlation between own trait position and judgments of ambiguous information about others and (b) neither depends on the mediating role of affect. However, encoding and informational processes do theoretically differ in the range of target conditions under which they are most likely to occur. On the one hand, informational effects could presumably come into play whenever the target information has unclear implications for the trait dimension of judgment and therefore could operate when the descriptive implications are completely irrelevant to that trait. On the other hand, effects of differential accessibility on trait encodings of information about others should occur only when the implications of ambiguous target information are directly relevant to the accessible concept (Higgins, 1989; Wyer \& Srull, 1989). Thus, one way to distinguish informational effects from encoding effects is that the latter of these should occur for ambiguous information but not for irrelevant information.

\section{Overview of Experiments}

Experiment 1, which was conducted in the domain of sociability, was designed as an initial exploration of the possibly different roles of the self in judgment of unambiguous and ambiguous information about others. Experiment 2 also focused on sociability and was designed to replicate crucial features of Experiment 1 while testing an alternative interpretation of that study. Experiments 3 and 4, conducted in the domains of independence and patience, respectively, tested certain critical predictions of our theoretical framework for different trait dimensions.

\section{Experiment 1: Judgments of Sociability}

In this experiment, subjects who differed in their own position with regard to sociability judged the sociability and favorableness of behaviors with high, low, or ambiguous implications for that trait. Half of the subjects formed favorableness judg-

\footnotetext{
${ }^{3}$ As Wyer and Srull (1989) have noted, one important ambiguity surrounding this literature is that nearly all studies have failed to unconfound extremity of self-definition from the self-rated importance of the relevant trait, which typically are positively correlated (but see Fuhrman \& Funder, 1991). To address this issue, the independent and interactive effects of these two variables on trait judgments is examined in the present research.
} 
ments of these behaviors prior to making their sociability judgments, with the order reversed for the remaining subjects.

This design was based on a similar procedure and logic used by Judd and Harackiewicz (1980) in the attitude domain. They noted that subjects' evaluative reactions to a given stimulus can vary with respect to individual differences in the extremity as well as the temporary salience of those reactions. Paralleling their manipulation in the attitude domain, the salience of evaluative reactions to the target behaviors during the sociability judgment task was assumed to be greater for those subjects who first rated these statements on the favorableness dimension. To the extent that favorableness and sociability are positively correlated for most subjects, accentuation theory predicts that increasing the salience of these affective reactions should produce an overall polarization effect. That is, both high-sociability and low-sociability subjects should judge high behaviors higher (and low behaviors lower) in sociability when evaluative judgments come first (a Judgment Order $\times$ Target interaction).

Measuring evaluative judgments also allowed us to determine the strength of affective reactions for each subject. Highly sociable subjects were expected to be more extreme in their evaluative reactions to the target behaviors. Accentuation theory predicts that these subjects should form more polarized judgments of the behaviors, regardless of judgment order (an Own Position $\times$ Target interaction).

The predicted Own Position $\times$ Target interaction is also consistent with social judgment theory (Sherif \& Hovland, 1961). However, the two theories make different predictions for analyses of covariance (ANCOVAs) and regression analyses of the sociability judgments of these behaviors. Social judgment theory predicts a relation between own position and judgments of the unambiguous stimuli even when the variance in affective reactions are factored or partialed out. Accentuation theory predicts that removing the variance associated with these reactions will reduce, or even eliminate, this relation. Accentuation theory further suggests that judgments should correlate strongly with the extremity of subjects' evaluative reactions to these stimuli, even when the variance associated with own position is removed.

Finally, a different pattern of results is predicted for judgments of the ambiguous behaviors. Because neither informational nor encoding mechanisms depend on the mediating role of affect, the effect of own position on judgments of these stimuli should not be contingent on manipulation of affective salience. Therefore, only a main effect for subject type is predicted: Highly sociable subjects should judge these behaviors as more sociable than subjects of low sociability, regardless of judgment order. Moreover, we predicted the effect of own position on judgments of these behaviors to remain, even when differences due to subjects' affective reactions are removed using ANCOVA and regression analyses.

\section{Method}

\section{Subjects and Design}

A total of 119 introductory psychology students from the University of Illinois participated in partial fulfillment of a course requirement. Subjects who varied in their self-reported sociability were asked to form favorableness and sociability judgments of short behavioral statements that represented high, low, or ambiguous levels of that trait. The order of these two judgment tasks was reversed for half of the subjects. The design therefore factorially combined two between-subjects variables, judgment order (evaluative judgments before vs. after sociability judgments) and level of own position (high vs. low), with one withinsubjects factor, type of target behaviors (high, low, and ambiguous implications for sociability). Other relevant variables included as part of the regression analyses were (a) the extremity of subjects' affective reactions to the target behaviors and (b) their ratings of the importance of sociability to the self. Inclusion of the latter measure was included in light of its centrality to the construct of self-schematicity as postulated by Markus (1977).

\section{Procedure}

Subjects were informed that they would be participating in a number of tasks designed to investigate how people form judgments of various types of social stimuli. They completed an initial task in which they were asked to form favorableness or sociability judgments of 16 behaviors. In the evaluative task, subjects rated each behavior on a scale that ranged from not at all faworable (0) to extremely favorable (10). Subjects in the sociability task rated each behavior on a scale that ranged from not at all sociable (0) to extremely sociable (10). After completing the initial task, subjects were given a second booklet and judged the same 16 behaviors (presented in reverse order) with respect to the remaining judgmental dimension (i.e., favorableness or sociability). Subjects worked on each task separately, and they were given no information as to the nature of any forthcoming tasks.

\section{Target Stimuli}

Fourteen of the target behaviors were relevant to sociability, 12 of which were specifically constructed to have implications for that trait that were either unambiguously low (e.g." "didn't try to get acquainted with many of the neighbors in the dorm") or high (e.g., "tries to go to at least one party every week"). Ambiguous items in this experiment were operationally defined as those statements that could imply either low or high levels of sociability. Further operational characteristics of ambiguous statements were that (a) they should be midrange items and (b) they should have substantially greater variance than unambiguous items. Two behaviors were specifically developed for this purpose and seemed intuitively likely to convey a desire either to facilitate, or to avoid, socializing with others: "installed a telephone answering machine in his/her apartment" and "decided to join the Peace Corps." Finally, two behaviors were included that had direct implications for a trait other than sociability, that is, studiousness (e.g., "worked hard to achieve good grades in college"). For half of the subjects, the entire sequence of behaviors rated across the two judgment tasks was reversed. Because none of the results were contingent on this counterbalancing of order, all of the analyses are collapsed over this variable.

\section{Self-Questionnaire}

After the second rating task, subjects completed a 20 -min distracter task (unrelated to the current investigation) that was followed by 12 questions that measured subjects' beliefs about their own sociability. The questionnaire consisted of general and specific statements pertaining to sociability (e.g., "In general, I always enjoy having the chance to meet new people"). For each item, subjects indicated how closely their own behavior matched the behavioral statement by circling a number on a scale that ranged from not at all descriptive of me $(0)$ to very descriptive of me (10). On the second page, subjects were presented with a number of bipolar dimensions, one of which pertained to a sociability-shyness dimension. Between these two attributes, $11 X$ s appeared, and subjects were instructed to "circle the one $X$ that best represents how you view yourself on this dimension." Finally, following a proce- 
dure used by Markus (1977), subjects were asked to indicate how important each of these dimensions was to them by circling a number on a scale that ranged from not at all important $(0)$ to very important $(10)$.

\section{Reliability and Validity of Self-Questionnaire}

Data from the 212 subjects who participated in Experiments 1 and 2 are combined here to describe properties of the questionnaire, which were quite consistent across studies. A measure of subjects' sociability was derived from nine items which loaded highly (.50 or better) on the first factor of a principal-components analysis. A composite index of subjects' sociability scores was calculated by taking the mean of these items (all scored on a $0-10$ scale) after reverse scoring, where appropriate.

The reliability of the composite index, estimated by Cronbach's alpha, was high, .91 . The distribution of scores on the index ranged from 0.89 to 9.67 , with a median of 6.00 . To help establish the validity of the scale, the 93 subjects in Experiment 2 were also administered Eysenck's revised extraversion scale (Eysenck, Eysenck, \& Barrett, 1985). The correlation between subjects' composite sociability score and the average score of the items from Eysenck's scale that pertain specifically to sociability (as opposed to impulsiveness) was high $(r=.72)$. Across both studies, there was a moderately high correlation between subjects' composite sociability scores and importance ratings $(r=.56)$, replicating previous studies by Markus and colleagues (e.g., Markus et al., 1985).

Because the self-questionnaire was administered after subjects' judgments of the target behaviors, there was a possibility of reactance of scores to experimental manipulation. To investigate this possibility, relevant analyses of variance (ANOVAs) were conducted using subjects' sociability as the dependent variable. No significant main effects or interactions were found (all $F \mathrm{~s}<1.0$ ), indicating that experimental conditions did not differentially affect self-ratings. Therefore, differences in sociability ratings of the target stimuli as a function of experimental condition cannot be attributed to changes in subjects' beliefs about their own sociability.

\section{Results}

\section{Preliminary Analyses}

Selection of target behaviors. Mean sociability ratings of the 14 behaviors relevant to sociability ranged from 1.78 to 8.71 , with standard deviations ranging from 1.31 to 2.36. Analyses of the behaviors confirmed our expectations about the nature of the behavioral items. The standard deviation of the two items that were classified a priori as ambiguous with respect to sociability ( $S D s=2.36$ and 2.31 ) were substantially higher than the standard deviations of the items designed to convey unambiguous implications for sociability, most of which were 1.60 or lower. Final criteria for selecting target stimuli for subsequent analyses were as follows: (a) items with mean ratings above 7.5 and standard deviations below 2.0 were classified as high behaviors, (b) items with mean ratings below 3.5 and standard deviations below 2.0 were classified as low behaviors, and (c) items with means between 4.5 and 6.5 and standard deviations above 2.30 were classified as ambiguous behaviors. Use of these criteria resulted in classification of 4 high-sociability behaviors, 5 low-sociability behaviors, and 2 ambiguous behaviors.

Index of evaluative extremity. For the regression analyses, a measure of individual differences in the extremity of subjects' affective reactions was adopted from a similar measure used by
Judd and Harackiewicz (1980). For each subject, a difference score was computed by subtracting the mean of the evaluative rating of low behaviors from that of the high behaviors. This difference score (hereinafter termed evaluative extremity) had a median of 3.41. The reliability of this index (following Allen \& Yen, 1979 , pp. 208-211) was acceptable, .81. This difference score correlated positively with subjects' own sociability $(r=$ .44), indicating that subjects of high sociability were more extreme in their evaluative reactions to the unambiguous target stimuli. The correlation between extremity of own position and affective reaction parallels results found in the attitude literature (cf. Judd \& Johnson, 1984). Results of an ANOVA indicated that scores on this index were not contingent on whether evaluative judgments from which it was computed occurred before or after sociability judgments $(F<1.0)$.

\section{Analyses of Variance}

Subjects whose composite sociability scores were above the median were classified as high subjects $(M=7.52)$, and those with scores below the median were classified as low subjects $(M=4.49) .^{4}$ The dependent variables of interest were the favorableness and sociability ratings of the different targets. These were analyzed separately using $2 \times 2 \times 3$ repeated measures ANOVAs with own position (high vs. low) and judgment order as between-subjects variables and type of target behavior (high, low, and ambiguous) as a within-subjects variable.

Evaluative judgments. Overall, subjects rated the high behaviors more favorably $(M=7.58)$ than the low behaviors $(M=$ $4.17)$, with the ambiguous behaviors rated between these two extremes $(M=5.43), F(2,230)=121.85, p<.001$. As suggested by the correlation between the evaluative extremity index and own position, high subjects were more extreme in their evaluation of the unambiguous stimuli $(M s=8.13$ vs. 3.53$)$ than were low subjects $(M \mathrm{~s}=7.03$ vs. 5.02$)$. High subjects also rated the ambiguous behaviors more favorably than did low subjects $(M \mathrm{~s}=5.85$ vs. 5.02$)$. The two-way interaction implied by this pattern was significant, $F(2,230)=17.29, p<.001$, and was not contingent on judgment order $(p>.25)$.

Sociability judgments. Sociability judgments of the three classes of target behaviors are displayed in Table 1 . Because the method for classifying the three types of behaviors was on mean sociability ratings, the main effect for target was expected and was not particularly meaningful. Of more interest were two highly significant interactions: (a) an Own Position $\times$ Target interaction, $F(2,230)=11.35, p<.001$, and (b) a Judgment Order $\times$ Target interaction, $F(2,230)=6.13, p<.003$. Because our theoretical framework suggests that these interactions reflect, in part, different processes for ambiguous and unambiguous targets, separate ANOVAs were conducted on these two classes of stimuli.

\footnotetext{
Across the four experiments reported in this article, the mean score of this latter group of subjects was around or somewhat above the midpoint of the scale. Therefore, it was unclear whether these subjects should be most accurately labeled as moderate or low. Note, however, that the overall pattern of judgments by subjects whose score fell in the lower third of the distribution generally resembled that of the low subjects. Therefore, our use of the label appeared to be justified.
} 
Results for the ambiguous targets were consistent with predictions of non-affective-based processes. High subjects judged these behaviors as more sociable than did low subjects $(M \mathrm{~s}=$ 6.86 vs. 5.86$), F(1,115)=9.15, p<.003$. More important, this pattern did not depend on whether evaluative judgments occurred before or after these judgments $(F<1.0)$. Additional evidence for our interpretation of this effect is provided by ANCOVAs and regression analyses.

A different pattern, consistent with predictions of accentuation theory, emerged for the unambiguous behaviors. First, judgments of the high and low behaviors were more polarized for high than for low subjects. That is, high subjects judged the high behaviors as more sociable $(M \mathrm{~s}=8.60 \mathrm{vs} .8 .10)$ and the low behaviors as less sociable ( $M \mathrm{~s}=2.62$ vs. 3.29 ). This was reflected in an Own Position $\times$ Target interaction, $F(1,115)=$ $10.32, p<.002$. Note that this was true for both judgment orders. Second, both groups of subjects were more polarized in their judgments of the high and low targets when favorableness judgments occurred before sociability judgments $(M s=8.67 \mathrm{vs}$. $2.59)$ than when they did not $(M s=8.06$ vs. 3.30). This was reflected in a Judgment Order $\times$ Target interaction, $F(1,115)=$ $13.26, p<.001$.

In combination, these two interactions are consistent with the hypothesis that sociability judgments of unambiguous behaviors are mediated by both individual differences in the extremity and manipulations of the salience of subjects' evaluative reaction to the targets. Moreover, the absence of a threeway (Own Position $\times$ Target $\times$ Judgment Order) interaction ( $p>$ $.15)$ suggests that the selected and manipulated variance in affective reactions had additive polarizing effects on sociability judgments of the high and low behaviors.

\section{Analyses of Covariance}

ANCOVAs were expected to provide further evidence of the different processes underlying judgments of the unambiguous and ambiguous behaviors. Accentuation theory predicts that differences in subjects' judgments of the unambiguous behaviors will be related to affective extremity rather than own position, but social judgment theory makes the opposite predic-

Table 1

Sociability Judgments of Target Behaviors as a Function of Own Position and Judgment Order: Experiment 1

\begin{tabular}{lll}
\hline \multirow{2}{*}{$\begin{array}{c}\text { Judgment } \\
\text { order/sociability } \\
\text { implications }\end{array}$} & \multicolumn{2}{c}{ Subject's own position } \\
\cline { 2 - 3 } & High & Low \\
\hline $\begin{array}{l}\text { Evaluative judgments first } \\
\text { High }\end{array}$ & 9.03 & \\
Low & 2.25 & 8.23 \\
Ambiguous & 6.98 & 2.99 \\
& & 5.67 \\
Trait judgments first & 8.12 & \\
High & 3.02 & 8.01 \\
Low & 6.71 & 3.52 \\
Ambiguous & & 6.00 \\
\hline
\end{tabular}

Note. Sociability scale ranges from not at all sociable $(0)$ to extremely sociable (10). tion. Both encoding and information-based processes predict strong effects of own position to emerge for judgments of the ambiguous behaviors, after affective reactions to these behaviors have been factored out. Analyses revealed that when subjects' evaluative ratings of the unambiguous behaviors were factored out, the highly significant Own Position $\times$ Target interaction for trait judgments was now only marginally significant $(p>.07)$. In contrast, the main effect for judgments of the ambiguous behaviors remained significant, $F(1,112)=4.82$, $p<.03$, after factoring out subjects' different affective reactions to these behaviors.

Another analytic approach is to dichotomize subjects (using median splits) on the basis of their scores on the evaluative extremity index and then compare these two groups in their sociability judgments of the unambiguous and ambiguous behaviors after factoring out the effects of own position. For unambiguous behaviors, the relevant Subject (high vs. low affective response) $\times$ Target interaction was highly significant, $F(1$, 115 ) $=16.45, p<.04$, after own position (which itself was not significant, $p>.20$ ) was covaried out. In contrast, analyses of the ambiguous behaviors revealed no effect for affective extremity $(p>.20)$ after own position was covaried out. In this case, the covariate of own position did, as predicted, account for a significant proportion of variance, $F(1,115)=8.80, p<.005$.

\section{Regression Analyses}

Regression analyses were performed to further explore the independent and interactive effects of the three individual-difference variables in this study (i.e., own position, affective extremity, and importance) on sociability judgments of the three types of target behaviors. Because initial analyses revealed no significant increment in squared multiple correlations when quadratic and interaction terms were added to the two equations, these terms were not included in the analyses we report here.

The unique effects of each of these three predictors on judgments of the three types of targets are given by the second-order partial correlations, as shown in Table 2. The implications of these analyses were similar to those of the ANCOVAs. The only significant partial correlation for the ambiguous targets was own position. The partial correlations between the evaluative index and ratings of the high and low behaviors were significant and in opposite directions, showing an effect of evaluative reactions to the behaviors when controlling for subjects' own sociability. However, own position did not significantly correlate with ratings of these two types of behaviors when the variance it shared with the evaluative index was removed. Finally, selfrated importance of sociability had no effects on judgments of either the unambiguous or ambiguous targets.

\section{Analyses of Irrelevant Behaviors}

Unlike the behaviors that had direct implications for sociability, analyses of the behaviors that had direct implications for studiousness ( $M=4.92$ ) revealed no significant effects of any of the measured or manipulated variables in this study (all $p \mathrm{~s}>$ .10). These null results are of interest for two reasons. First, they rule out the possibility that the differences in the way that 
Table 2

Second-Order Partial Correlations Among Subject Variables and Sociability Judgments of Target Behaviors: Experiment 1

\begin{tabular}{llcc}
\hline & \multicolumn{3}{c}{ Sociability implications } \\
\cline { 2 - 4 } Subject variable & High & Low & Ambiguous \\
\hline Own position & .08 & -.13 & $.15^{*}$ \\
Affective extremity & $.16^{*}$ & $-.26^{* *}$ & .07 \\
Importance & .08 & .02 & .05 \\
\hline
\end{tabular}

${ }^{*} p<.05$, one-tailed test. ${ }^{* *} p<.01$, one-tailed test.

high and low subjects judged ambiguous behaviors were simply due to differential use of the response scale for midrange behaviors. Second, although the irrelevant behaviors are similar to the ambiguous behaviors in that they are relatively undiagnostic with respect to their level of sociability, these data show that low diagnosticity was not in itself a sufficient condition for the effect of own position to occur.

\section{Discussion}

Sociability judgments of both unambiguous and ambiguous behaviors were contingent on how subjects defined themselves with respect to that trait, but these effects appeared to be guided by different processes. Judgments of unambiguous behaviors were not directly mediated by the extremity of subjects' own sociability per se, as predicted by social judgment theory (Sherif \& Hovland, 1961). Rather, judgments of these stimuli were most directly mediated by the polarizing effect of their affective reaction to the target behaviors, as suggested by accentuation theory (Eiser \& Stroebe, 1972; Tajfel, 1957). However, own position did have a significant positive effect on judgments of ambiguous behaviors, even when the variance associated with subjects' different affective reactions to these stimuli was removed.

There are at least two processes that could account for the pattern of data that emerged for judgments of the ambiguous behaviors. When subjects attempted to form a sociability judgment of these targets, they may have used general knowledge about the characteristics of the self and their acquaintances to derive the descriptive implications of these stimuli. Alternatively, to the extent that differences in subject sociability are related to differences in the chronic accessibility of that trait, high subjects may have had a greater likelihood of spontaneously categorizing the behavior as sociable than did low subjects.

Effects of own sociability were observed for ambiguous behaviors, but not for the irrelevant behaviors (which, like the ambiguous behaviors, were relatively undiagnostic with respect to their level of sociability). This provides at least some evidence that effects on ambiguous targets may have involved encoding, as distinguished from informational, processes. However, because differences in chronic accessibility were not directly measured in this study, our data do not permit definitive conclusions to be reached in this regard. Nevertheless, our data clearly show that self-rated sociability exerted a significant ef- fect on judgments of the ambiguous behaviors that occurred independently of subjects' affective reactions to them. This was not the case for the unambiguous behaviors.

\section{Experiment 2: Testing an Alternative Interpretation}

Although Experiment 1 provides a reasonably clear account of the processes underlying judgments of ambiguous and unambiguous behaviors, it is subject to a potentially serious flaw. In that experiment, measures of affective extremity were obtained from the same behaviors that were judged for sociability, and therefore this study failed to obtain an independent assessment of affective reaction. Because such an assessment was not made, alternative interpretations can be raised regarding the possible effects of simply recalling favorableness ratings and using these directly in judgments of sociability.

\section{Method}

To investigate this issue, we conducted a second experiment $(N=$ 93), which followed the procedure of the evaluation-first condition in Experiment 1 with one important variation. Whereas half of the subjects formed evaluative judgments of the same stimuli that were later rated for sociability (as in Experiment 1), the remaining subjects formed evaluative judgments of behaviors that, although relevant to the domain of sociability, were different from those that were later judged in the trait-rating task. If the results obtained in Experiment 1 are due to an artifact of subjects forming evaluative and trait judgments of the same stimuli, the polarization effects demonstrated in that experiment should disappear when the stimuli judged in the evaluative and trait judgment task differ. However, our framework suggests that similar polarization effects should be obtained regardless of this factor.

\section{Results and Discussion}

As in Experiment 1, the contingency between subjects' own level of sociability and their judgments of the unambiguous and ambiguous behaviors were analyzed using ANOVAs, ANCOVAs, and regression analyses. The results of these analyses in all cases were nearly identical to those obtained in Experiment 1. Specifically, these data replicated the overall Own Position $\times$ Target interaction found in Experiment $1, F(2,170)=10.26, p<$ .001 . That is, high subjects judged high and ambiguous behaviors as more sociable than did low subjects, but this pattern was reversed for the low behaviors. Moreover, the pattern of partial correlations (shown in Table 3) provided a near-exact replication of the results from Experiment 1 and again indicated that the effect of subjects' own level of sociability on judgments of the unambiguous and ambiguous behaviors are mediated by different processes. None of these results, however, were contingent on whether sociability judgments were preceded by evaluative judgments of the same or different sociable-relevant behaviors (all $F \mathrm{~s}<1.0$ ), thus demonstrating that the polarization effects demonstrated in Experiment 1 were not an artifact of subjects forming sociability and evaluative judgments of the same stimuli.

\footnotetext{
${ }^{5}$ We also manipulated whether subjects formed judgments of behaviors (e.g., "went to three parties a week") or of persons ("Bob went to three parties a week"). This variable was included to explore the possi-
} 
Table 3

Second-Order Partial Correlations Among Subject Variables and Sociability Judgments of Target Behaviors: Experiment 2

\begin{tabular}{lllc}
\hline & \multicolumn{3}{c}{ Sociability implications } \\
\cline { 2 - 4 } Subject variable & High & Low & Ambiguous \\
\hline Own position & .11 & -.09 & $.23^{* *}$ \\
Affective extremity & $.36^{* *}$ & $-.18^{*}$ & .11 \\
Importance & .00 & -.05 & -.08 \\
\hline
\end{tabular}

${ }^{*} p<.05$, one-tailed test. ${ }^{* *} p<.01$, one-tailed test.

\section{Path Analysis}

Another approach to testing our theoretical framework is to model the relations among our predictor and criterion variables using path-analytic techniques. Our theoretical perspective provides predictions about which classes of models should adequately predict the covariance structure among the relevant variables in our study and which classes of models should be inadequate. To test these predictions, we conducted a series of analyses using the LISREL VI (Jöreskog \& Sörbom, 1984) program for analyzing structural equations. The six variables we analyzed included the three predictor variables (subject sociability, evaluative extremity, and importance indexes) for which we have reported separate regression analyses. For all analyses, the correlation matrix for the combined subjects of Experiments 1 and $2(N=212)$ was analyzed using the maximum likelihood estimation procedure. Because we analyzed the correlation matrix, the path coefficients are analogous to standardized regression coefficients for predicting one variable from another.

Because each of the three criterion variables consisted of the same type of judgment (sociability) made for similar types of behavioral targets within the same time period, we assumed that these three measures would be intercorrelated. To test this assumption, we conducted several analyses under the constraint that the errors for the three criterion variables were uncorrelated. None of the identified models (with $5 d f \mathrm{~s}$ or more) provided an adequate fit. Thus, for all subsequent analyses we used three free parameters to model the correlated errors among the three criterion variables. In addition, because the evaluative extremity index was based on favorableness ratings of the high and low targets, it stands to reason that the errors for this index should be correlated with errors for sociability judgments of these targets. Thus, two paths were added to account for these correlated errors.

Figure 1 presents a path diagram that is consistent with our a priori model of how the predictor variables influence one another as well as influence sociability ratings of the different targets under the constraints discussed above. Our three pre-

bly different processes that may underlie judgments of generic behaviors versus persons (cf. Wyer \& Lambert, in press). Aside from a general tendency for persons to be rated as higher in sociability than behaviors, however, none of the results of this study were contingent on this factor. dictor variables were significantly correlated with one another. Theoretically, it is reasonable to assume that both evaluative and importance indexes are driven by conceptions of one's own sociability. These relations are depicted by the two paths leading from the sociability index to the importance and evaluative indexes. A supporting line of evidence for this assumption is that adding a direct path between the evaluative and importance indexes does not lead to a significantly better fit $(p>.10$ for this path).

Our a priori model implied that only three additional parameters would be needed to provide an adequate fit to the judgment data. These were (a) a positive relation between ratings of the high sociability targets and evaluative extremity, (b) a negative relation between ratings of the low sociability targets and evaluative extremity, and (c) a positive relation between the ambiguous targets and self-rated sociability. This model provided a good fit to the data, $\chi^{2}(5, N=212)=6.63, p=.25$, with all three of these path coefficients significant $(p<.05)$ and none of the other path coefficients between predictor and criterion variables significant $(p>.10)$. In summary, the path analyses support a general theoretical framework in which the effects of self-reported sociability on judgments of unambiguously low and high targets are mediated through evaluative reactions to these stimuli, but the effects of self-reported sociability on judgments of ambiguous stimuli are not.

\section{Experiment 3: Judgments of Independence}

As depicted in Figure 1, our theoretical framework provides a straightforward summary of the role of the self in judgments of others in the domain of sociability. However, it would be desirable to demonstrate that the implications of our model generalize to other trait dimensions. In this regard, note that in an unpublished study by Markus and Fong (1979; reported in Markus \& Smith, 1981) highly independent subjects formed more polarized judgments of unambiguously independent and dependent targets compared with subjects whose self-definitions were less extreme. Although Markus and Fong interpreted their findings within the framework of social judgment theory, our model suggests that these results occurred through accentuation processes rather than through self-anchoring mechanisms. Because Markus and Fong did not assess subjects' evaluative reactions to the targets, their results do not distinguish between these two possibilities. Experiment 3 was conducted in the domain of independence to resolve this ambiguity and therefore establish the generality of our model across two trait dimensions.

\section{Method}

\section{Subjects and Design}

The basic design and procedure of Experiment 3 were very similar to Experiment l's. A total of 82 introductory psychology students who differed in their self-reported independence were asked to form independence and evaluative judgments of persons whose behaviors varied in their implications for that trait. The design included two betweensubjects variables (own position and judgment order) and one withinsubjects variable (type of target behaviors). 


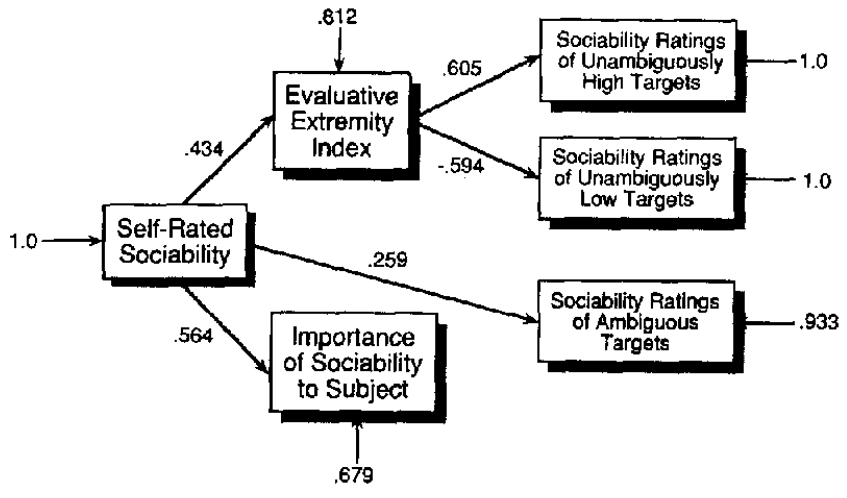

Figure 1. Results of path analysis concerning the relation between subject variables (self-rated sociability, importance, and evaluative extremity) and sociability judgments of high, low, and ambiguous targets. (All paths shown are significant at $p<.05$ )

\section{Behavioral Items}

Sixteen of the target stimuli were specifically constructed to have implications for independence that were unambiguously high (e.g., "When it came to making decisions about his carrer, Sam always trusted his own instincts") or low ("Before casting his vote for president of the student body, Joe checked with his friends to see who they were voting for ${ }^{n}$ ). In addition, four targets were included that had unambiguously high or low implications for a different trait, intelligence. We also attempted to construct items that were ambiguous with respect to independence. However, we were unsuccessful in doing so, because these items had properties that were similar to high, rather than ambiguous, stimuli.

\section{Self-Questionnaire}

As in Experiments 1 and 2, the self-questionnaire consisted of two sections. The first section included 12 behavioral statements (e.g., "On the whole, I'd say that I'm pretty independent"). The second section presented subjects with the three bipolar trait dimensions used by Markus (1977; independent-dependent, individualist-conformist, and leader-follower), for which subjects were asked to indicate their own position as well as the importance of the dimension to the self. Because principal-components analyses again suggested a one-factor solution, four highly loading items were combined into a composite independence index, which had a high reliability (Cronbach's $\alpha=89$ ). The distribution of scores ranged from 3.50 to 10.00 , with a median of 7.63. There was again a high correlation between scores on this composite index and importance ratings $(r=.57)$. As in Experiment 1 , no significant effect of judgment order on self-ratings emerged $(p>.25)$.

\section{Results and Discussion}

\section{Preliminary Analyses}

Targets receiving the four highest and four lowest independence ratings were classified as high and low behaviors, respectively. Trait ratings of the four high targets ranged from 9.20 to 8.66 , with standard deviations between .96 and 1.59 ; ratings of the low targets ranged from 1.46 to 2.02 , with standard deviations between 1.28 and 1.54 . The measure of evaluative extremity was computed in the same way as in Experiments 1 and 2, that is, by subtracting subjects' evaluations of the low behaviors from those of the high behaviors. As in the case of sociability, scores on this index correlated positively with own position $(r=$ $.31, p<.002$ ).

\section{Analysis of Variance}

Subjects whose composite independence scores were above or below the median were classified as high or low subjects, respectively. Independence and evaluative judgments of the high and low targets were analyzed separately using a $2 \times 2 \times 2$ repeated measures ANOVA, with own position and judgment order as between-subjects variables and target type as a withinsubjects variable.

The results of Experiments 1 and 2 suggest that both evaluative and trait ratings of the target stimuli would be more extreme for the high subjects. This was in fact the case. For evaluative judgments, all subjects rated the high targets more favorably than the low targets, but this pattern was again more extreme for high subjects $(M \mathrm{~s}=7.57$ vs. 2.82$)$ than for low subjects $(M \mathrm{~s}=6.72$ vs. 3.49$), F(1,78)=14.86, p<.001$. The parallel pattern for trait judgments emerged; that is, high subjects were more extreme in their trait judgments of the high and low targets $(M \mathrm{~s}=9.15$ vs. 1.59$)$ than were low subjects $(M \mathrm{~s}=$ 8.75 vs. 1.96$), F(1,78)=4.38, p<.04$. Unlike in Experiment 1 , however, a significant Judgment Order $\times$ Target interaction for the trait judgments failed to emerge in this experiment $(p>15)$.

\section{Analyses of Covariance}

Given our conceptualization of the results of Experiments 1 and 2, our expectation was that the Own Position $\times$ Target interaction that pertained to subjects' independence judgments of the high and low targets was a result of differences in their affective reactions to these stimuli, rather than a direct use of own position as a judgmental anchor. The ANCOVAs confirmed this expectation, showing that the Own Position $\times$ Target interaction for trait judgments was rendered nonsignificant $(F<1.0)$ when differences in subjects' evaluative reactions were factored out. Similarly, in the 2 (high vs. low affective extremity) $\times 2$ (high vs. low target) ANCOVA, the two-way interaction was significant, $F(1,79)=15.06, p<.001$, whereas the covariate of own position was not $(p=.14)$. Finally, as in Experiments 1 and 2 , subjects' own independence had no significant effect on the independence judgments of persons whose behaviors had unambiguously high or low implications for a different trait (i.e., intelligence). ${ }^{6}$

\footnotetext{
${ }^{6}$ Regression analyses yielded results almost identical to those obtained in Experiments 1 and 2, with only two exceptions. Although the ANCOVAs showed no effect of own position on judgments of the unambiguous behaviors, the partial correlation of own position and judgments of the low targets did attain significance in this analysis $(r=$ $-.19, p<.05$ ), providing some evidence for a self-anchoring effect (contrast) for this class of stimuli. Second, a significant partial correlation emerged between the self-rated importance of independence and trait ratings of the low behaviors $(r=.24)$, a pattern for which we could generate no plausible explanation. Because none of the other studies in this article provided any hint of a similar pattern, it seemed best to regard this finding as spurious.
} 
Taken together, these data replicate the effects demonstrated by Markus and Fong (1979), showing more polarized independence judgments of unambiguous stimuli by subjects who defined themselves extremely with respect to that trait. As in the domain of sociability, however, these effects were primarily due to the accentuating effect of affective response rather than direct use of own position as a judgmental anchor.

\section{Experiment 4: Implications for Universally Evaluated Traits}

An important aspect of our theoretical framework is that reliable effects of own position on judgments of unambiguous information should arise only to the extent that there is a systematic relation between extremity of own position and affective reactions toward these stimuli. Although this was indeed the case for the trait dimensions of sociability and independence, this relation may not exist for all traits. This issue is directly relevant to a distinction that Sherman, Chassin, Presson, and Agostinelli (1984) have raised between universally and variably evaluated dimensions. Universally evaluated dimensions, on the one hand, are those for which all persons (regardless of own position) have similar evaluative reactions to stimuli implying high or low levels of the dimension. Variably evaluated dimensions, on the other hand, are those for which high and low levels of the dimension evoke different evaluative responses across judges. Experiments $1-3$ indicate that both sociability and independence are variably evaluated dimensions.

Our model predicts that the contingency between own position and trait judgments of unambiguous behaviors should be reliable whenever the trait dimension is variably evaluated. However, these effects should not occur when the trait dimension is universally evaluated. This is because the kinds of affective response to behaviors having implications for such traits should be similar regardless of own position, and therefore the polarization effects that emerged in the domain of sociability and independence should fail to emerge for universally evaluated dimensions.

These different predictions for variably and universally evaluated traits apply only to judgments of unambiguous stimuli. According to our model, individual differences in the judgment of ambiguous behaviors are (unlike unambiguous behaviors) driven by own position independently of perceivers' affective response to these stimuli. Therefore, the relation between self-definition and judgments of ambiguous behaviors should occur regardless of whether the trait dimension is variably or universally evaluated.

Experiment 4 focused on the trait dimension of patience, a dimension that Sherman et al. (1984) have empirically demonstrated to be universally evaluated. We therefore expected subjects' self-reported patience to be unrelated to their evaluation of behaviors having implications for this trait. Of more importance, however, are the predictions of our model for the effects of own position on trait judgments of ambiguous and unambiguous stimuli. Highly patient subjects should judge ambiguous behaviors as more patient than should low subjects (an own position main effect), replicating the results found in Experiments 1 and 2. However, the strong Own Position $\times$ Target interactions found in trait judgments of unambiguous stimuli in the domain of sociability and independence should not emerge in this study.

\section{Method}

The method of Experiment 4 was very similar to that used in Experiments 1 and 3. Subjects were 80 undergraduates from the University of South Carolina who participated in partial fulfillment of a course requirement. Subjects were asked to form trait and favorableness judgments of persons whose behaviors varied in their implications for patience. Judgment order was manipulated between subjects, and type of target (high, low, or ambiguous) was manipulated within subjects. Included in the design were two subject variables: subjects' self-reported patience and their evaluative extremity with respect to the patience dimension.

\section{Behavioral Items}

The target stimuli were designed to have implications for patience that were unambiguously high (e.g., "Pat didn't mind waiting for the bus, even though he knew that the next bus wouldn't come for at least half an hour"), low ("Richard yelled at the taxi driver because he thought he wasn't driving fast enough"), or ambiguous (e.g., "After the waiter told Paul that it would take 2 hours to get a seat, Paul left the restaurant to call his friends about a change in plans").

\section{Self-Questionnaire}

Because of time constraints in administration of the experiment, the self-questionnaire was completed immediately after the judgment task. Subjects rated themselves with respect to five patience-related statements (e.g., "On the whole, I'd say I'm quite patient") and also rated how important the trait of patience was to them. In a principal-components analysis, five of the items loaded highly on the first component; thus, these items were combined to create a measure of self-rated patience. The measure had satisfactory reliability (Cronbach's $\alpha=82$ ), with the distribution of scores ranging from 1.0 to 9.4 and a median of 5.8. As in Experiments 1-3, no significant effects of judgment order on self-ratings emerged $(p>.25)$.

\section{Results}

\section{Preliminary Analyses}

Mean ratings for the seven items classified as low behaviors ranged from 0.68 to 3.02 , with a mean of 1.97. Mean ratings for the seven items classified as high behaviors ranged from 7.75 to 8.52 , with a mean of 8.02 . Standard deviations for the two types of unambiguous stimuli averaged 1.52. Three of the remaining items were classified as ambiguous behaviors; these had midrange values (4.47-6.22) and larger standard deviations (2.182.32) than any of the unambiguous targets.

The evaluative extremity index was computed by subtracting each subject's mean evaluative rating of the low targets from that of the high targets. Unlike the strong and significant correlation between own position and evaluative extremity that emerged for the variably evaluated dimensions of sociability and independence, the correlation between subjects' own patience and evaluative extremity was only marginally significant $(r=.21, p=.06)$. This is consistent with earlier evidence by Sherman et al. (1984) that patience is universally rather than variably evaluated. However, because this correlation is some- 
what larger than we expected, the ANOVAs reported below (which treat own position and evaluative extremity as orthogonal variables) are supplemented by appropriate ANCOVAs.

\section{Analyses of Variance}

In these analyses, own position and evaluative extremity were dichotomized (using median splits) and entered as variables in four-way ANOVAs performed on evaluative and patience ratings separately. In the analyses, own position (high vs. low), evaluative extremity (high vs. low), and judgment order (trait vs. evaluative ratings first) were between-subjects variables, and target (low, high, and ambiguous) was the only within-subjects variable.

The four-way ANOVA conducted on evaluative ratings revealed only two significant effects. First, the main effect of target, $F(2,71)=548.18, p<.001$, reflected a strong liking for the high targets $(M=7.62)$, less so for the ambiguous targets $(M=6.15)$, and least for the low targets $(M=3.15)$. The only other significant effect was the interaction between evaluative extremity and target, which merely reflects the selection criterion for assignment to low and high evaluative extremity groups. Finally, the fact that own position had no effects whatsoever on evaluative judgments (all $F s<1.0$ ) lends additional support to the assertion that patience is universally evaluated.

The four-way ANOVA conducted on patience ratings produced four statistically significant results, the first of which was simply an artifactual main effect of target produced by the selection criterion used for the three levels of target. The other significant effects were all two-way interaction effects with the target variable and included (a) a Judgment Order $\times$ Target interaction, $F(2,144)=16.30, p<.001$; (b) an Evaluative Extremity $\times$ Target interaction, $F(2,144)=15.17, p<.001$; and (c) an Own Position $\times$ Target interaction, $F(2,144)=6.63, p<.01$. These three interactions are displayed in the three panels of Figure 2. Following our a priori theoretical distinctions between unambiguous and ambiguous behaviors, we investigated these interactions separately for the two classes of target stimuli.

Unambiguous behaviors. Analyses conducted on the unambiguous targets revealed that the interactions involving judgment order and evaluative extremity were highly significant ( $p s<.001)$, reflecting the pattern predicted by accentuation theory. As shown in the left panel of Figure 2, patience judgments were more polarized for all subjects when these ratings were preceded by evaluative judgments than when they were not (a Judgment Order $\times$ Target interaction). Similarly, the center panel shows that patience judgments of both the high and low behaviors were more polarized for subjects whose evaluative reactions to the target stimuli were extreme (an Evaluative Extremity $\times$ Target interaction). As predicted for the universally evaluated trait of patience, the strong Own Position $X$ Target interaction that emerged in Experiments 1-3 (in which high subjects formed more polarized judgments of the high and low behaviors) did not emerge in this study. That is, the difference between patience judgments of the high and low behaviors for high subjects $\left(M_{\text {diff }}=5.97\right)$ was nearly identical to that for the low subjects $\left(M_{\text {diff }}=6.06\right), F \mathrm{~s}<1.0$.

Finally, as shown in the right panel of Figure 2, ratings of both the high and low targets were lower for the high patience subjects, resulting in a significant main effect of own position, $F(1,72)=4.84, p<.05$. Although this effect is relatively small, it indicates an overall contrast effect on judgments of these behaviors, which is not contingent on affective reactions. The fact that contrast occurred for both high and low behaviors is at odds with social judgment theory (Sherif \& Hovland, 1961) but is more compatible with other, alternate conceptualizations of anchoring effects (e.g., Upshaw, 1969).

Ambiguous behaviors. The three-way ANOVA performed on the ambiguous target revealed, as predicted, a significant main effect of own position on judgments of these behaviors, such that high subjects judged ambiguous behaviors as more patient than did low subjects $(M \mathrm{~s}=5.53$ vs. 4.81$), F(1,72)=7.51, p<$ .01 (see right panel of Figure 2). Also as predicted, this effect was not contingent on either judgment order (replicating the effects found in the domain of sociability) or on evaluative extremity (both $p s>.15$ ). ${ }^{7}$

\section{Analyses of Covariance}

Although the correlation between evaluative extremity and own position was not significant, the classification of subjects in the analyses reported above did not produce a completely orthogonal design. To verify that the effects reported above for own position and evaluative extremity are independent of one another, we conducted analyses that paralleled those reported above but covaried out either evaluative ratings of the targets or self-reported patience.

The pattern of significance reported above was unchanged when the relevant ANCOVAs were performed. In particular, these analyses provided strong support for three main predictions of our theoretical framework: (a) significant positive effect of own position on judgments of ambiguous behaviors after factoring out evaluative reactions to these behaviors, (b) accentuating effect of manipulated salience and extremity of subjects' evaluative reactions to the unambiguous behaviors that occurred after factoring out own position, and finally (c) no polarization effect related to own position (i.e., the lack of an Own Position $\times$ Target interaction) because of the universally evaluated nature of patience.

\section{Discussion}

We have demonstrated that the effects of own position on trait judgments of unambiguous stimuli depend on whether the trait dimension is variably or universally evaluated. This find-

\footnotetext{
${ }^{7}$ The only other effect to emerge was a main effect of evaluative extremity, such that the ambiguous target was judged lower in patience by subjects who were high in evaluative extremity, $F(1,72)=4.06, p<$ .05 , a result that was not contingent on either self-reported patience or on judgment order. This finding can be explained within the context of accentuation theory in light of the fact that subjects high in evaluative extremity judged these stimuli more unfavorably than those low in evaluative extremity. For our purposes, however, the point to emphasize is that the effects of own position on judgments of the ambiguous stimuli occurred, as in the domain of sociability, independently of subjects' affective reaction to this information.
} 


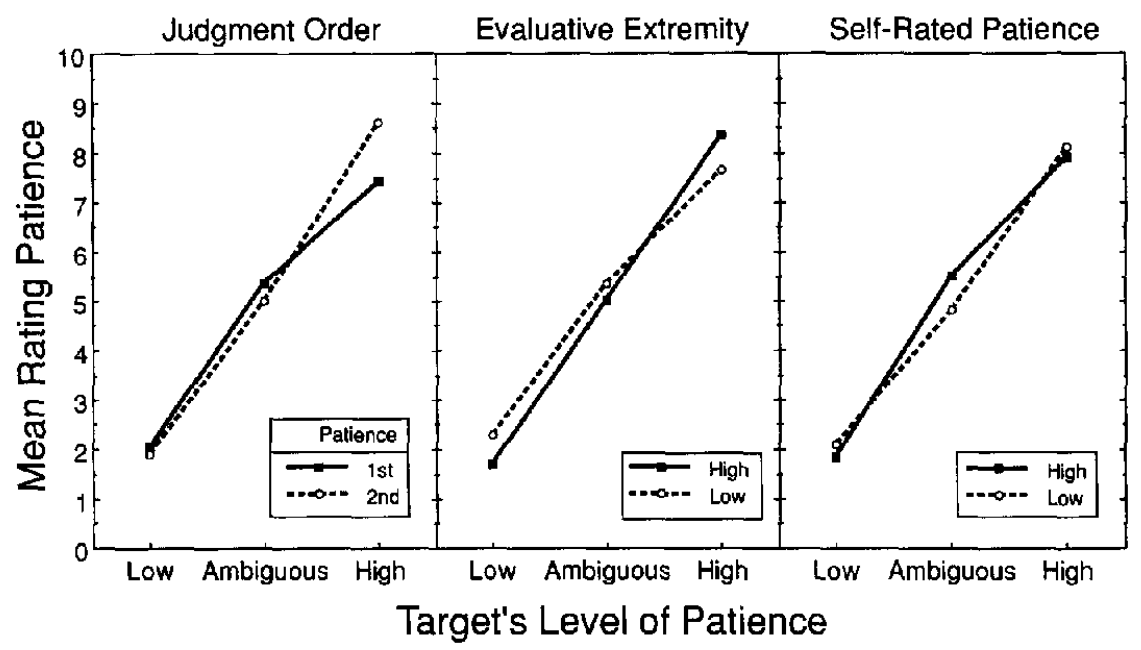

Figure 2. Patience ratings of the behaviors implying low, ambiguous, and high levels of patience as a function of judgment order (left panel), evaluative extremity (center panel), and self-rated patience (right panel).

ing may help to resolve some of the inconsistent results characteristic of this literature. Affect-based polarization effects of the sort that occur for variably evaluated traits appear to be a rather strong and highly replicable effect, and therefore one may expect a reliable contingency between own position and trait judgments of unambiguous stimuli for such traits. However, the effects of own position on judgments of unambiguous stimuli for universally evaluated traits occur only through the use of the self as a judgmental anchor. Although this effect did emerge for the trait of patience, the strength of this effect appears to vary considerably across trait dimensions and indeed may not occur at all for certain traits.

In this regard, note that in two studies that focused on the trait dimension of masculinity-femininity, weak or unreliable effects of the self on trait judgments of persons with unambiguously masculine or feminine characteristics were obtained (Markus et al., 1985; Park \& Hahn, 1988). The null results reported in those studies may be a consequence of that dimension being one that is universally evaluated. In particular, extremely masculine and moderately masculine subjects in the Markus et al. study did not differ in their evaluative reactions to a target who engaged in unambiguously masculine behaviors, suggesting that masculinity is a universally evaluated trait. The unreliable effect of own position on trait ratings of unambiguous stimuli found in these studies is consistent with what our theoretical model would predict for this type of trait dimension.

\section{General Discussion}

Our experimental results provide several insights into the general processes by which individual differences in self-definition may play a role in how unambiguous and ambiguous information about persons and behaviors is judged. These results support the following conclusions:

1. When the target information implied unambiguously high or low levels of the trait dimension, individual differences in trait judgments were mediated largely by differences in subjects' affective reaction to them. When the extremity of these reactions was reliably correlated with own position (as in the case of the variably evaluated traits of sociability and independence), there was greater polarization of judgment for subjects whose own positions were extreme. When extremity of own position and affective responses were not significantly correlated (as in the case of the universally evaluated trait of patience), polarization effects were not related to own position.

2. Subjects' own position had a positive effect on trait judgments of ambiguous information, even when the variance associated with affective reactions to these stimuli was removed. These effects occurred regardless of whether the trait was variably or universally evaluated and were moderated by either encoding or informational processes.

3. Relatively weak support was found for the direct use of own position as a judgmental anchor in the judgment of unambiguous stimuli. When such evidence was obtained, it was in the form of a general contrast effect, which emerged most strongly in the domain of patience. We found no evidence for assimilation effects for unambiguous stimuli.

\section{The Role of Affect in Judgments of Others}

Social and cognitive psychologists have long believed that the effect of own attitude on the opinions of others is driven by the same principles of assimilation and contrast that guide the judgment and perception of evaluatively neutral stimuli in the psychophysical domain (cf. Sherif \& Hovland, 1961). Those few studies in the trait domain that have examined the effects of the self on judgments of unambiguous information about others (e.g., Markus \& Fong, 1979) have adopted a similar perspective. Together with previous research by Judd and colleagues in the attitude domain (Judd \& Harackiewicz, 1980; Judd \& Johnson, 1984), our findings indicate that the effect of the self on judgments of others is driven considerably more by affect-based polarization effects than by these anchoring mechanisms. Al- 
though general contextual effects are undoubtedly a pervasive and robust phenomenon in many judgmental settings, the widespread assumption that self-knowledge functions as a judgmental anchor in the ratings of other persons and behaviors may need to be reevaluated.

Although the kind of polarization effects demonstrated in this article was originally conceptualized within a broader theory of human judgment initially proposed by Tajfel (1957), it also possible to view these effects within the affect-as-information framework proposed by Schwarz and Clore (1987). Schwarz and Clore generally argued that one's affective state or experience at the time of judgment may often be used as a source of information in its own right, guiding how the target stimulus should be rated. Although their research pertains mostly to the informational value of mood states rather than positive or negative reactions to specific stimuli, the general view of affect as information that they postulate seems applicable to the present context as well. For example, when subjects are asked to judge the sociability of the behavior "rarely talks to others in the hallway," they may be somewhat uncertain of its value along the sociable-unsociable dimension, even though it clearly falls below the midpoint of that underlying dimension. To the extent that unfavorable reactions are generally associated with low levels of sociability, a very negative reaction to this stimulus may serve as additional information that the stimulus indeed represents a low level of that trait. An analogous process pertains to judgments of behaviors representing high levels of that trait.

\section{Processes Underlying Judgments of Unambiguous and Ambiguous Stimuli}

Our results show quite conclusively that the self can have strong effects on the judgment of both unambiguous and ambiguous information about others, but these effects involve rather different processes. The general notion that judgments of these two classes of behaviors involve different processes has also been articulated by Herr et al. (1983) in the priming domain. Herr et al. theorized that judgment of ambiguous stimuli is largely an identification task (e.g., "Is it a member of category $X$ or $Y$ ?"), but judgments of unambiguous information primarily involve a comparison of the value of the stimulus to a given context or stimulus that is salient at the time of judgment. In support of this reasoning, Herr et al. showed that priming semantic categories can, under certain conditions, have positive (assimilative) effects on the judgment of ambiguous stimuli (presumably through encoding mechanisms), but only negative (contrast) effects on the judgment of unambiguous information.

Although there is some theoretical overlap between our conceptualization and that of Herr et al. (1983), at least two important distinctions should be made. First, the main theoretical question in our research is how existing differences in subjects' self-concept can influence trait judgments of other persons and behaviors. This differs from the primary issue investigated by Herr and his colleagues, namely, how judgments of target stimuli may be influenced by subtle activation of semantic concepts (e.g., ferocity of animals) that have little or no direct relevance to subjects' self-concepts.
Second, in our research, differences in the judgment of unambiguous stimuli were driven primarily by differences in subjects' affective reaction to them (accentuation). The effects found by Herr et al. (1983), however, did not fit the polarization pattern characteristic of affect-based processing but rather reflected general contrast effects of the sort described by Upshaw (1969), which is generally how Herr et al. framed their findings. The positive effects of own position on judgments of ambiguous stimuli in this article (Experiments 1,2, and 4) may well have involved the type of encoding processes described by Herr et al. and others. However, an alternative informational interpretation of our results for ambiguous stimuli is possible and involves a process very different than the one that presumably typically occurs in priming studies. Nevertheless, our research clearly reinforces the general point made by Herr et al., namely, that different theoretical considerations and processes are likely to come into play, depending on the descriptive implications of the target stimulus for the dimension of judgment.

Indeed, one of the more intriguing aspects of our research is the possibly different roles of own position and chronic accessibility on the judgment of different classes of target stimuli. Direct use of self-relevant information as a basis for judging others can theoretically occur over a wide range of target information and could even occur when no trait-relevant information is available (as in the false-consensus paradigm). Encoding processes presumably cannot occur under these conditions because there is simply no relevant information to be encoded. Instead, encoding processes should be limited primarily to ambiguous information that is relevant to the trait construct (cf. Higgins, 1989). By measuring different aspects of the self and varying the type of information being judged, a clearer picture of the different processes by which the self can affect judgments of others should emerge.

\section{References}

Allen, M. J, \& Yen, W. M. (1979). Introduction to measurement theory. Belmont, CA: Wadsworth.

Bargh, J. A. (1982). Attention and automaticity in the processing of self-relevant information. Journal of Personality and Social Psychology, 43, 425-436.

Bargh, J. A. (1984). Automatic and conscious processing of social information. In R. S. Wyer, Jr., \& T. K. Srull (Eds.), Handbook of social cognition (Vol. 3, pp. 1-43). Hillsdale, NJ: Erlbaum.

Bargh, J. A. (1989). Conditional automaticity: Varieties of automatic influence in social perception and cognition. In J. S. Uleman \& J. A. Bargh (Eds.), Unintended thought (pp. 3-51). New York: Guilford Press.

Bargh, J. A., Bond, R. N., Lombardi, W., \& Tota, M. E. (1986). The additive nature of chronic and temporary sources of chronic accessibility. Journal of Personality and Social Psychology, 50, 869-878.

Berscheid, E., \& Walster, E. H. (1978). Interpersonal attraction. Reading, MA: Addison-Wesley.

Eiser, J. R. (1986). Social psychology: Attitudes, cognition, and social behavior. Cambridge, England: Cambridge University Press.

Eiser, J. R., \& Stroebe, W. (1972). Categorization and social judgment. San Diego, CA: Academic Press.

Eiser, J. R., \& van der Plight, J. (1984). Accentuation theory, polarization, and judgment of attitude statements. In J. R. Eiser (Ed.), Attitudinal judgment (pp. 43-63). New York: Springer-Verlag.

Eysenck, S. G., Eysenck, H. J., \& Barrett, P. (1985). A revised version of 
the psychoticism scale. Personality and Individual Differences, 6, 2129.

Freud, S. (1956). Further remarks on the defense neuropsychoses. In J. Rieviere (Ed), Collected papers of Sigmund Freud (Vol. 1, pp. 155182). London: Hogarth Press. (Original work published 1896).

Fuhrman, R. W., \& Funder, D. C. (1991). Individual differences in personality and in the processing of self-relevant information. Manuscript submitted for publication.

Heider, F. (1958). The psychology of interpersonal relations. New York: Wiley.

Helson, H. (1964). Adaptation-level theory. New York: Harper \& Row.

Herr, P. M., Sherman, S. J., \& Fazio, R. H. (1983). On the consequences of priming: Assimilation and contrast effects. Journal of Experimental Social Psychology, 19, 323-340.

Higgins, E. T. (1989). Knowledge accessibility and activation. In J. S. Uleman \& J. A. Bargh (Eds.), Unintended thought (pp. 75-123). New York: Guilford Press.

Higgins, E. T., \& Bargh, J. A. (1987). Social cognition and social perception. Annual Review of Psychology, 38, 369-425.

Higgins, E. T., \& King, G. (1981). Accessibility of social constructs: Information processing consequences of individual and contextual variability. In N. Cantor \& J. F. Kihlstrom (Eds.), Personality, cognition, and social interaction (pp. 69-122). Hillsdale, NJ: Erlbaum.

Higgins, E. T., King, G. A., \& Mavin, G. H. (1982). Individual construct accessibility and subjective impressions and recall. Journal of Personality and Social Psychology, 73, 35-47.

Higgins, E. T., Rholes, W. S., \& Jones, C. R. (1977). Category accessibility and impression formation. Journal of Experimental Social Psychology, 13, 141-154.

Jöreskog, K. G., \& Sörbom, D. (1984). LISREL VI: Analysis of linear structural relationships by maximum likelihood, instrumental variables, and least squares methods. Mooreshead, IN: Scientific Software.

Judd, C. M., \& Harackiewicz, J. M. (1980). Contrast effects in attitude judgment: An examination of the accentuation hypothesis. Journal of Personality and Social Psychology, 38, 390-398.

Judd, C. M., \& Johnson, J. T. (1984). The polarizing effects of affect. In R. Eiser (Ed.), Attitudinal judgment (pp. 65-82). New York: Springer.

Kelly, G. A. (1955). The psychology of personal constructs. New York: Morton.

Marks, G., \& Miller, N. (1987). Ten years of research on the false-consensus effect: An empirical and theoretical review. Psychological Bulletin, 102, 72-90.

Markus, H. (1977). Self-schemata and processing information about the self. Journal of Personality and Social Psychology, 35, 63-78.

Markus, H., \& Fong, G. (1979). The role of the self on other perception. Unpublished manuscript, University of Michigan.

Markus, H., \& Smith, J. M. (1981). The influence of self-schemas on the perception of others. In N. Cantor \& J. F. Kihlstrom (Eds.), Personality, cognition, and social interaction (pp. 233-262). Hillsdale, NJ: Erlbaum.

Markus, H., Smith, J., \& Moreland, R. L. (1985). Role of the self-concept in perception of others. Journal of Personality and Social Psychology, 49, 1494-1512.
Markus, H., \& Zajonc, R. (1985). The cognitive perspective in social psychology. In G. Lindzey \& E. Aronson (Eds.), The handbook of social psychology (3rd ed., Vol. 1, pp. 137-230). New York: Random House.

Newcomb, T. M. (1961). The acquaintance process. New York: Holt, Rinehart, \& Winston.

Park, B., \& Hahn, S. (1988). Sex-role identity and the perceptions of others. Social Cognition, 6, 61-87.

Petty, R. E., \& Cacioppo, J. T. (1981). Attitudes and persuasion: Classic and contemporary approaches. Dubuque, IA: William C. Brown.

Schwarz, N., \& Clore, G. L. (1987). How do I feel about it? The informative function of affective states. In K. Fiedler \& J. Forgas (Eds), Affect, cognition, \& social behavior (pp. 44-62). Toronto, Canada: Hogrefe.

Segal, Z. (1988). Appraisal of the self-schema construct in cognitive models of depression. Psychological Bulletin, 2, 147-162.

Sherif, M., \& Hovland, C. I. (1961). Social judgment: Assimilation and contrast effects in communication. New Haven, CT: Yale University Press.

Sherif, M., \& Sherif, C. W. (1967). Attitude as the individual's own categories: The social judgment-involvement approach to attitude and attitude change. In C. W. Sherif \& M. Sherif (Eds.), Attitude, ego-involvement, and change (pp. 105-139). New York: Wiley.

Sherif, M., Taub, D., \& Hovland, C. (1958). Assimilation and contrast effects in anchoring stimuli on judgments. Journal of Experimental Psychology, 55, 150-155.

Sherman, S. J., Chassin, L., Presson, C. C., \& Agostinelli, G. (1984). The role of the evaluation and similarity principles in the false consensus effect. Journal of Personality and Social Psychology, 47, 12441262.

Srull, T. K., \& Wyer, R. S. (1980). Category accessibility and social perception: Some implications for the study of person memory and interpersonal judgment. Joumal of Personality and Social Psychology, 38, 841-856.

Suchman, E. A. (1950). The intensity component in attitude and opinion research. In S. A. L. Guttman, E. A. Suchman, P. A. Lazarsfeld, S. A. Star, \& J. A. Clausen (Eds.), Studies in social psychology in World War II: Vol. 4. Measurement and prediction (pp. 213-276). Princeton, NJ: Princeton University Press.

Tajfel, H. (1957). Value and the perceptual judgment of magnitude. Psychological Review, 64, 192-204.

Upshaw, H. S. (1969). The personal reference scale: An approach to social judgment. In L. Berkowitz (Ed.), Advances in experimental social psychology (Vol. 4, pp. 315-371). San Diego, CA: Academic Press.

Wyer, R. S., \& Lambert, A. J. (in press). The role of trait constructs in psychological research and theory: An historical perspective. In $\mathbf{P}$. Devine, T. Ostrom, \& D. Hamilton (Eds.), Social cognition: Contributions to classical issues in social psychology. New York: Springer-Verlag.

Wyer, R. S., \& Srull, T. K. (1989). Human cognition in its social context. Hillsdale, NJ: Erlbaum.

Received July 30, 1990

Revision received April 4, 1991 Accepted June 27, 1991 